\title{
Infección por Rhodotorula mucilaginosa en trasplante alogénico de progenitores hematopoyéticos, reporte de un caso
}

Rhodotorula mucilaginosa infection in hematopoietic stem cell transplantation setting, case report

\author{
- Mauricio Chaparro1, Marcela Estupiñán', Germán Camacho 2,4 , Gloria Uribe \\ Unidad de Oncohematología y Trasplante de Progenitores Hematopoyéticos, Fundación HOMI-Hospital de la Misericordia. \\ Unidad de Infectología Pediátrica, Fundación HOMI-Hospital de la Misericordia. \\ Servicio de Hematología Especial, Laboratorio Clínico, Fundación HOMI-Hospital de la Misericordia. \\ ${ }^{4}$ Universidad Nacional de Colombia.
}

\section{Resumen}

Se describe el caso de una paciente de 9 años de edad con infección por Rhodotorula mucilaginosa asociada a catéter venoso central, con anemia aplásica adquirida severa después de trasplante alogénico de progenitores hematopoyéticos de donante idéntico emparentado.

Palabras clave: Rhodotorula, anemia aplásica, huésped inmunocomprometido.

\begin{abstract}
Infection by Rhodotorula spp. is recognized as an emerging infection in the last two decades especially in immunocompromissed patients. This yeast has been described as not very virulent although it can produce uncomplicated systemic infection in blood or even potentially fatal infections such as endocarditis or ventriculitis. Its isolation is associated with central venous catheters. There are only a few cases reported in recipients of hematopoietic transplantation.
\end{abstract}

Key words: Rhodotorula, hematopoietic stem cell transplantation, aplastic anemia, idiopathic.

\section{Introducción}

La Rhodotorula spp. es una levadura de la familia Cryptococcus ubicua en el medio ambiente, en la flora humana y en diversos alimentos y bebidas', que produce colonias mucoides de color rojizo. Se ha reconocido como una infección emergente en las dos últimas décadas, especialmente en pacientes inmunosuprimidos². Esta levadura se ha descrito como poco virulenta, aunque puede producir infecciones sistémicas, como fungemias no complicadas, e infecciones potencialmente fatales, como meningitis, endocarditis, ventriculitis ${ }^{3}$. Se ha asociado a la presencia de catéteres venosos centrales ${ }^{4}$; en la literatura están reportados solo unos pocos casos en receptores de trasplante hematopoyético ${ }^{5}$.

\section{Caso}

Se describe el caso de una paciente de sexo femenino de 9 años de edad con diagnóstico de anemia aplásica adquirida severa con celularidad en la médula ósea menor al 5\%, sin fragilidad cromosómica en el cariotipo con mitomicina $C$, sin malformaciones esqueléticas; se descartó patología infecciosa desencadenante, hemoglobinuria paroxística nocturna y no hay claros antecedente tóxicos, aunque vive en área rural de zona agrícola; tiene un hermano 100\% idéntico en la evaluación de los antígenos de histocompatibilidad.

Recibe trasplante alogénico de progenitores hematopoyéticos de donante emparentado, es 
hospitalizada en habitación con sistema de filtración de aire de alta eficiencia; desde el ingreso se inserta catéter tunelizado de larga duración por vía venosa cefálica izquierda. De acuerdo con las guías institucionales, recibe profilaxis antiinfecciosa con aciclovir, trimetoprim sulfametoxazol y fluconazol, profilaxis para enfermedad injerto contra huésped con ciclosporina y metotrexate. Recibe médula ósea alogénica que contiene 2,4 x 108 células totales nucleadas $/ \mathrm{kg}$ de peso, con incompatibilidad mayor $A B O$ (receptor $\mathrm{O}+$ /donante $\mathrm{A}+$ ). En el hemograma del día +14 se encuentra rápido aumento del recuento de leucocitos hasta $1.160 / \mathrm{mm}^{3}$, el extendido de sangre periférica revela imágenes sugestivas de acúmulos de levaduras sin evidencia de leucocitos (ver figura 1), no tiene fiebre ni deterioro del estado general, inicia en forma empírica terapia combinada con caspofungina y voriconazol. A las 24 horas de terapia antifúngica el recuento de leucocitos en el contador celular regresa a 0. Los hemocultivos revelan el crecimiento de Rhodotorula mucilaginosa, inicia manejo específico con anfotericina B deoxicolato (no se disponía en el país de la formulación liposomal); en los hemocultivos de control persiste el crecimiento de la levadura, se descartó endocarditis, se retiró el catéter tunelizado como parte del manejo de infección fúngica invasiva. La paciente evoluciona satisfactoriamente con muy lenta recuperación de la hematopoyesis, presenta injerto de los neutrófilos al día +70 e injerto plaquetario al día +100 con quimerismo $100 \%$ de origen del donante. Es dada de alta sin complicaciones posteriores luego de 65 días de estancia hospitalaria,

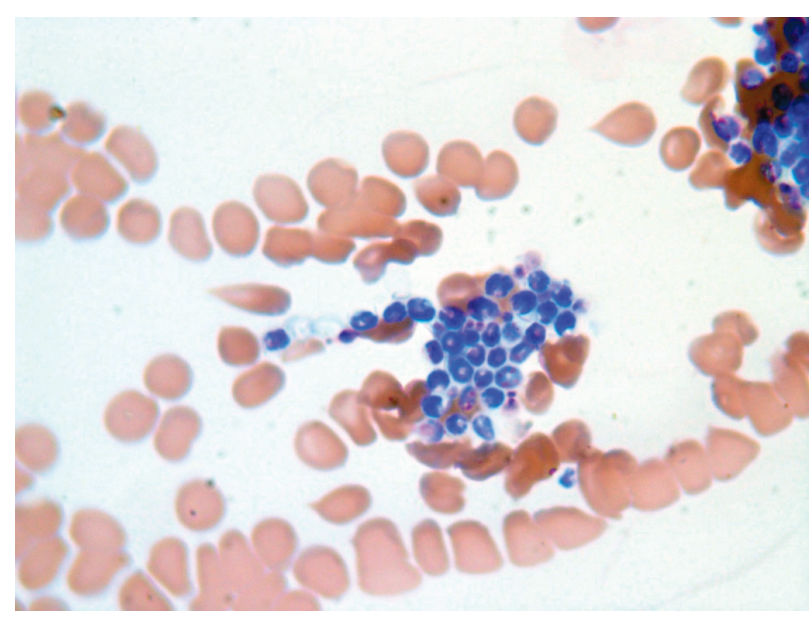

Figura 1. Sangre periférica, coloración de Wright 100x, acúmulos de levaduras, sin evidencia de leucocitos. sin infecciones ni hospitalizaciones posteriores y sin requerimiento transfusional; la lenta recuperación hematológica se atribuyó a la incompatibilidad $A B O$ más que al proceso infeccioso, no se documentaron complicaciones atribuibles al uso de la anfotericina B deoxicolato.

\section{Discusión}

Hay algunos casos previos de infección por Rhodotorula mucilaginosa descritos en pacientes pediátricos con enfermedades malignas ${ }^{6}$ y son muy pocos los reportados en el contexto del trasplante hematopoyético, la mayoría de ellos autólogos 5 .

Los agregados de levaduras confunden el contador celular simulando estructuras celulares, lo que ocasiona que se cuenten como leucocitos, generando falsos recuentos; el extendido de sangre periférica verifica la presencia de las estructuras micóticas.

Aun con una carga micótica importante evidenciada en el extendido de sangre periférica, la paciente no tiene clínica de infección sistémica grave; el hallazgo de baja virulencia ha sido descrito previamente?

Inicialmente considerada como un germen no patógeno, la Rhodotorula mucilaginosa es en la actualidad un patógeno emergente en población de riesgo; conforme con los casos publicados, esta población corresponde a pacientes inmunocomprometidos y que además tienen accesos venosos centrales, en tumores sólidos o neoplasias hematológicas, en los que se usan medicamentos citotóxicos y esteroides, y antibióticos de amplio espectro, y se les brinda nutrición parenteral ${ }^{2,3}$; también ha sido descrito en pacientes críticos en unidad de cuidados intensivos ${ }^{1}$.

La principal manifestación infecciosa suele ser el aislamiento en sangre, aunque se han descrito otras infecciones, como meningitis, prostatitis, ventriculitis, endocarditis ${ }^{3}$. La pancitopenia ha sido el momento de presentación usual de la infección sistémica por Rhodotorula en pacientes trasplantados ${ }^{8}$.

La relación de esta infección con el uso de catéteres se debe en parte a que este agente tiene gran capacidad de producir biofilm, similar al generado por las especies de Candida; además, los aislamientos provenientes de pacientes tienen una mejor capacidad de formar biofilm que los aislamientos provenientes del ambiente, por lo que el retiro del catéter es fundamental para el manejo 9,10. 
Los aislamientos de Rhodotorula son sensibles a anfotericina $B$, resistentes a equinocandinas y a fluconazol, y con sensibilidad variable a voriconazol y posaconazol11.

El tratamiento de elección para esta levadura es anfotericina $\mathrm{B}$; en este caso se utilizó anfotericina B deoxicolato. Aunque su actividad antimicótica es similar a las otras formas de anfotericina B, esta presentación tiene un riesgo mayor de toxicidad, agregada al efecto tóxico de la quimioterapia y al régimen preparativo; en el país ya se dispone de la preparación liposomal, con lo cual se sortean los efectos tóxicos relacionados con el tratamiento'12,13.

\section{Referencias}

1. Lunardi LW, Aquino VR, Zimerman RA, Goldani LZ. Epidemiology and outcome of Rhodotorula fungemia in a tertiary care hospital. Clin Infect Dis. 2006;43(6):e60-3.

2. Wirth F, Goldani LZ. Epidemiology of Rhodotorula: an emerging pathogen. Interdiscip Perspect Infect Dis. 2012;2012:465717.

3. Tuon FF, Costa SF. Rhodotorula infection. A systematic review of 128 cases from literature. Rev Iberoem Micol. 2008;25(3):13540.

4. Kiehn TE, Gorey E, Brown AE, Edwards FF, Armstrong D. Sepsis due to Rhodotorula related to use of indwelling central venous cetheters. Clin Infect Dis. 1992;14(4):841-6.

5. Mori T, Nakamura Y, Kato J, Sugita K, Kamei K, Okamoto S. Fungemia due to Rhodotorula mucilaginosa after allogeneic hematopoietic stem cell transplantation. Transpl Infect Dis. 2012;14(1):91-4.
6. Pascualotto GC, Copetti FA, Meneses CF, Machado AR, Brunetto AL. Infection by Rhodotorula sp. in children receiving treatment for malignant diseases. J Pediatr Hematol Oncol. 2005;27(4):232-3.

7. Forés R, Ramos A, Orden B, De la Iglesia A, Bautista G, Cabero $M$, et al. Rhodotorula species fungemia causes low mortality in hematopoietic stem-cell transplantation. A case report and review. Mycoses. 2012;55(3):e158-62.

8. Ramos $A$, Cabero $M$, Orden B. Fungemia por Rhodotorula mucilaginosa. Presentación de dos casos. Rev Esp Quimioter. 2012;25(1):76-8.

9. Nunes JM, Bizerra FC, Ferreira RC, Colombo AL. Molecular identification, antifungal susceptibility profile and biofilm formation of clinical and environmental Rhodotorula species isolates. Antimicrob Agents Chemother. 2013;57(1):382-9.

10. Kim HA, Hyun M, Ryu SY. Catheter associated Rhodotorula mucilaginosa fungemia in a immucompetent host. Infect Chemother. 2013;45(3):339-42.

11. Dieckema DJ, Petroelje B, Messer SA, Hollis RJ, Pfaller MA. Activities of available and investigational antifungal agents against Rhodotorula species. J Clin Microbiol. 2005;43(1):476-8.

12. Preney L, Théraud M, Guiguen C, Gangneux JP. Experimental evalulation of antifungal and antiseptic agents against Rhodotorula spp. Mycoses. 2003;46(11-12):492-5.

13. Arendrup MC, Boekhout $T$, Akova M, Meis JF, Cornely OA, Lortholary $\mathrm{O}$, et al. ESCMID and ECMM joint clinical guideline for the diagnosis and management of rare invasive yeast infections. Clin Microbiol Infect. 2014;20 Suppl 3:76-98.

14. García-Suárez J, Gómez-Herrus P, Cuadros JA, Burgaleta C. Epidemiology and outcome of Rhodotorula infection in haematological patients. Mycoses. 2011;54(4):318-24.

15. Simon MS, Somersan S, Singh HK, Hartman B, Wickes BL, Jenkins SG, et al. Endocarditis caused by Rhodotorula infection. J Clin Microbiol. 2014;52(1):374-8. 